\section{REFERENCES}

[1] J. E. Knowles, "Magnetic properties of individual acicular particles," IEEE Trans. Magn., vol. MAG-17, pp. 3008-3013, Nov. 1981.

[2] R. K. Waring, Jr., "Magnetic interactions in assemblies of singledomain particles. The effect of aggregation," J. Appl. Phys., vol. 38, pp. 1005-1006, 1967.

[3] L. Thurlings and W. Kitzen, "Switching behavior of interacting magnetic particles," IEEE Trans. Magn., vol. MAG-16, pp. 5355 , Jan. 1980.

[4] J. E. Knowles, "Coercivity and packing density in acicular particles," J. Magn. Magn. Mat., vol. 25, pp. 105-112, 1981.

[5] D. J. Craik and A. J. Harrison, "Magnetic field, potential, force, and energy calculations for permanent magnets," in Proc. 3rd Europ. Conf. Hard Magn. Mat., Amsterdam, 1974, pp. 33-36.
[6] E. Kneller and E. Köster, "Relation between anhysteretic and static magnetic tape parameters," IEEE Trans. Magn., vol. MAG13, pp. 1388-1390, Sep. 1977.

[7] J. E. Knowles, "Measurements on single magnetic particles," IEEE Trans. Magn., vol. MAG-14, pp. 858-860, 1978.

[8] D. M. Hiller, "High coercivity chromium dioxide," J. Appl. Phys, vol. 49 , pp. 1821-1822, Sep. 1978.

[9] A. H. Morrish and S. P. Yu, "Magnetic measurements on individual microscopic ferrite particles near the single-domain size," Phys. Rev., vol. 102, pp. 670-673, 1956.

[10] S. Umeki, H. Sugihara, Y. Taketomi, and Y. Imaoka, "The dependence of the coercivity on the volume packing fraction for magnetic particles," IEEE Trans. Magn., vol. MAG-17, pp. 3014-3016, Nov. 1981.

[11] L.F.G. Thurlings, private communication.

[12] R. M. Bozorth, Ferromagnetism. Princeton, NJ: van Nostrand, 1951, p. 846.

\title{
Perpendicular Stand-Still Recording in Co-Cr Films
}

\author{
T. WIELINGA, J. H. J. FLUITMAN, AND J. C. LODDER
}

\begin{abstract}
Some features of perpendicular recording were investigated by means of stand-still recording experiments. For this purpose several radio frequency (RF)-sputtered Co-Cr layers of two different compositions were used as media while a Permalloy single pole (SP) head was used as a write transducer. A magnetoresistive transducer (MRT) was utilized to read the recorded pattern. Further, an analytical model was developed enabling the magnetization induced by the head field in the recording medium to be calculated. Both calculations and experimental results show a sharpening of the transition, due to the demagnetizing field. For a good approximation it is sufficient to consider only the vertical head field component and vertical magnetization in the recording medium. This is a consequence of the well-developed perpendicular anisotropy and negligible in-plane remanence of the $\mathrm{Co}-\mathrm{Cr}$ layers. In addition the read-out signal is completely determined by the magnetic surface charges. The remanent magnetization in the recording medium and therefore the read-out amplitude is limited by demagnetization and consequently determined by the coercivity of the $\mathrm{Co}-\mathrm{Cr}$ layer. The medium noise of a dc-erased medium indicates magnetic structures of much larger dimensions than the size of the individual crystallites. This noise appears to be dependent on the saturation magnetization of the $\mathrm{Co}-\mathrm{Cr}$ medium. Activation of the single pole head by a homogeneous field results in a nonlinear behavior, caused by head saturation effects. This is supported by simple one-dimensional calculations of the head field.
\end{abstract}

Manuscript received March 17, 1982; revised October 21, 1982.

The authors are with Twente University of Technology, Department of Electrical Engineering, P.O. Box 217, 7500 AE Enschede, The Netherlands.

\section{INTRODUCTION}

QTAND-STILL recording, where the recording head is not Omoved during activation, offers the advantage of an analytical description of the recording process as shown in the case of longitudinal magnetic recording [1]. This concept is worked out for a recording medium with strong perpendicular anisotropy and the resulting model is used to predict the experimental results.

For these experiments sputtered $\mathrm{Co}-\mathrm{Cr}$ layers are used as a recording medium, a Permalloy single pole (SP) head is used for writing and a magnetoresistive transducer (MRT) for reading.

A Permalloy back layer between the substrate and the $\mathrm{Co}-\mathrm{Cr}$ medium has been proposed [2]. This layer greatly improves the efficiency of the head and recording characteristics [2], [3]. In our experiments however, this back layer has been omitted in order to reduce the number of experimental and theoretical problems. Our main purpose is to investigate the physical principles of perpendicular magnetic recording.

\section{A. The Co-Cr Recording Medium}

The $\mathrm{Co}-\mathrm{Cr}$ films were prepared by means of radio frequency (RF)-sputtering [4] from two different targets on silicon substrates, yielding the compositions 17 and 19 at\% $\mathrm{Cr}$. These 
films have a very high degree of $c$-axis orientation of the hexagonal close-packed (hcp) structure perpendicular to the film surface and the halfwidth angle $\Delta \theta_{50}$ of the 002-rocking curve is only about $1.5^{\circ}[5]$. As a consequence the films possess a strong positive crystal anisotropy [6]. Electron microscopy reveals a polycrystalline morphology and crystallites with a diameter of about $0.1 \mu \mathrm{m}$, depending on the film thickness, are present in the normal direction [5].

The hysteretic behavior of the $\mathrm{Co}-\mathrm{Cr}$ medium is measured by means of a vibrating sample magnetometer (VSM). Fig. 1(a) shows the perpendicular hysteresis loop, i.e., in the preferred direction of the magnetization. This hysteresis loop can be seen as the easy axis loop, sheared by the demagnetizing field. In the case of a thin magnetic film the demagnetizing factor in the perpendicular direction equals one (in this paper SI units will be used). It is assumed here that the size of the magnetic domains is small compared with the film thickness. The magnetization $M$, as measured by means of a VSM, is averaged over all domains and if a statistical orientation of their magnetization is assumed, $M$ may be considered as uniform on a scale of $1 \mu \mathrm{m}$ or larger. The total magnetic field $H_{\mathrm{tot}}$ is given by the sum of the uniform VSM field $H_{\mathrm{u}}$ and the demagnetizing field $H_{d}=-M$ in the perpendicular direction:

$$
H_{\text {tot }}=H_{u}-M \text {. }
$$

The saturation magnetization $M_{s}$, calculated from the saturation field of the perpendicular hysteresis loop according to (1) is $620 \mathrm{kA} / \mathrm{m}$ and is in reasonable agreement with the value of $670 \mathrm{kA} / \mathrm{m}$ as determined from the calibrated VSM voltage.

In Fig. 1(a) some minor loops are also shown. In approximation their horizontal trajectories are taken as lines of constant magnetization. This means that the magnetization is considered to be constant as long as the absolute value of the total field applied does not exceed the coercive field strength $H_{c}$ :

$$
\left|H_{\text {tot }}\right|=\left|H_{u}+H_{d}\right| \leqslant H_{c} .
$$

This rule will be applied to the case of stand-still recording. However, Fig. 1(a) shows that $M$ does not remain constant for the entire switch of $H_{\text {tot }}$ from $-H_{c}$ to $+H_{c}$. This deviation becomes manifest in the maximum remanent magnetization, as will be shown in Section V-C.

Fig. 1(b) shows the in-plane hysteresis loop, i.e., in the nonpreferred plane of magnetization. It is marked by a small remanence of about 4 percent and rotational behavior of the magnetization. From the saturation field an approximate value for the anisotropy field $H_{A}$ is found of about $560 \mathrm{kA} / \mathrm{m}$, corresponding very well with the value found by torque measurements $[6]$.

\section{B. The Basic Principles of the Proposed Model}

The next approximations and assumptions will be used in the model, as described by the theory in the Section III.

1) Only vertical components of the head field and the magnetization in the recording medium are considered. This is justified by the relatively large anisotropy field, compared with

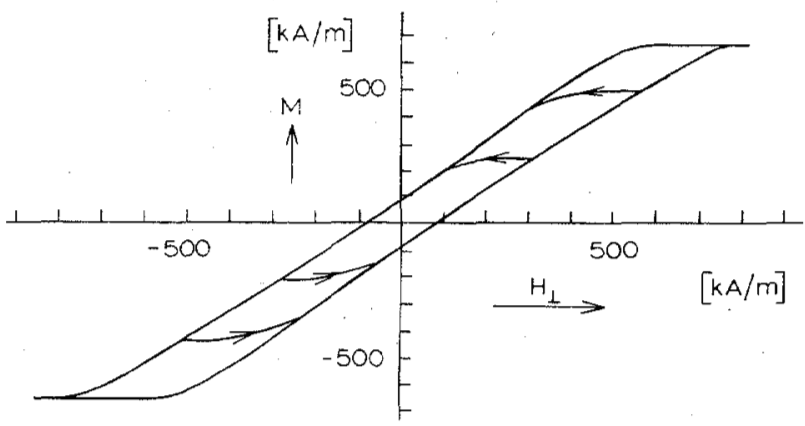

(a)

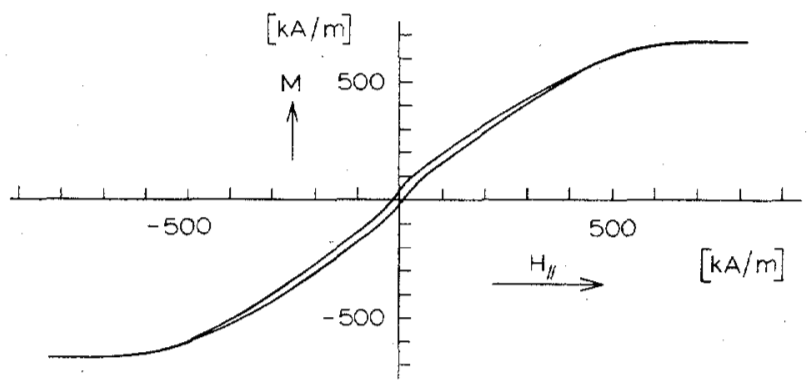

(b)

Fig. 1. Hysteresis loops of 17 at $\% \mathrm{Cr}$ alloyed $\mathrm{Ca}-\mathrm{Cr}$ film. (a) Perpendicular with some minor loops. (b) In-plane.

the horizontal components of the head field and the demagnetizing fields, and the small in-plane remanence.

2) $M$ is constant in the $z$-direction, or

$$
d M / d z=0
$$

for $-h / 2<z<h / 2$, where $h$ is the film thickness while the origin of the $z$-axis is in the middle of the film (see Fig. 2). This is justified by the fact that the magnetization is independent of the film thickness [6]. Furthermore the anisotropy field is relatively strong compared with the horizontal components of demagnetizing fields. Therefore only magnetic surface charges are considered in the calculations of the magnetic fields.

3) The magnetization $M$ will be considered as a continuous function of $x$. The diameter of the domains is much smaller than the dimensions of the heads used, resulting in an averaged contribution of all domains along the track width.

4) The transversal direction $y$ will be considered infinite, so the model is in fact two-dimensional.

5) The unsheared or intrinsic hysteretic behavior is considered to be valid locally. In a recording process the local values of $H_{\text {tot }}$ are usually different from those in the case of uniform fields and magnetization as described by (1). As usual the demagnetizing fields complicate the solution of the problem.

6) The influence of the magnetized recording medium on the head field will not be considered here, or in other words the magnetic charge image of the medium in the head is neglected. In addition there is no experimental evidence of this kind of interaction.

7) It is assumed that after switching off the head field the magnetization does not change in the recording layer. As 


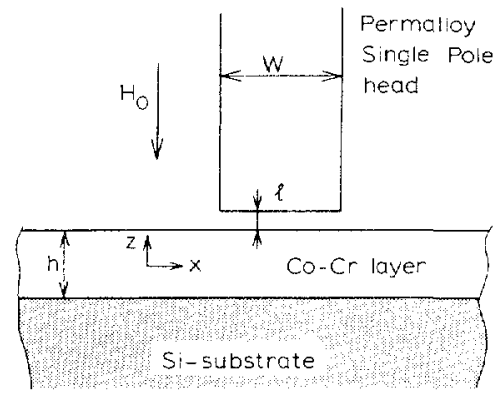

Fig. 2. Configuration for stand-still recording and coordinates used; $y$-axis is perpendicular to plane of drawing and dimensions along this axis are considered to be infinite; $H_{0}$ is homogeneous field activating the SP head of width $W$ at separation $i$ from $\mathrm{Co}-\mathrm{Cr}$ medium.

stated before [(2) with $H=0$ ] this is only true if $\left|H_{d}\right| \leqslant H_{c}$. This condition can be easily checked, because $H_{d}$ is known. In practice demagnetization occurs for high values of the head field (see Section V).

\section{Experimental Procedure}

In the recording process the resulting magnetization when applying a head field, is also determined by the initial state of magnetization. This will be made clear in the case of VSM measurements, where a perpendicular homogeneous field is applied (see Fig. 1(a)). After saturation of the sample by a positive field, the remanent magnetization $M_{r}$ at zero field follows from (2): $M_{r}=H_{c}$. Reversing and increasing the field results in a linear dependence of $M$ on the field: $M=\left(H_{u}+\right.$ $H_{c}$ ) where $H_{u} \leqslant 0$. If however the field is not reversed but again increased, $M$ will not change as long as $0 \leqslant H_{u} \leqslant 2 H_{c}$ but will increase linearly if $H_{u} \geqslant 2 H_{c}$ according to $M=\left(H_{u}-\right.$ $H_{c}$ ). From these considerations it follows that the resulting magnetic remanence will always be independent of the initial state of magnetization, if the applied field $H_{u}$ has been larger than $2 H_{c}$. In the recording process, the situation is much more complicated because of the nonuniformity of the head field and the demagnetizing field, but the initial state of magnetization remains important. To avoid this ambiguity in the experiments, the medium is saturated first in a strong (about $800 \mathrm{kA} / \mathrm{M}$ ) perpendicular field whereby the magnetization comes into one of the remanent states at $H=0$ as shown in Fig. 1(a). The SP head is now placed on the medium. The head to medium distance $l$ is measured by means of an optical interference method and the head is activated by a homogeneous field $H_{0}$ (see Fig. 2). This activating field, and therefore also the head field itself, is taken as antiparallel to the initial state of magnetization.

In this way the problem is linearized and an analytical solution is possible (see next section). For the read-out of the induced head print, a magnetoresistive transducer (MRT) is applied (see Fig. 3). (The same MRT was also used extensively for analysing recording head fields and offers the advantage of static reading [7]). The MRT to medium distance $s$ and its position along the $x$-axis are both measured by means of optical interference methods, giving a resolution of 0.05 and 0.1 $\mu \mathrm{m}$, respectively. The length, width, and thickness of the MRT are 50,2 , and $0.05 \mu \mathrm{m}$, respectively. The MRT is only

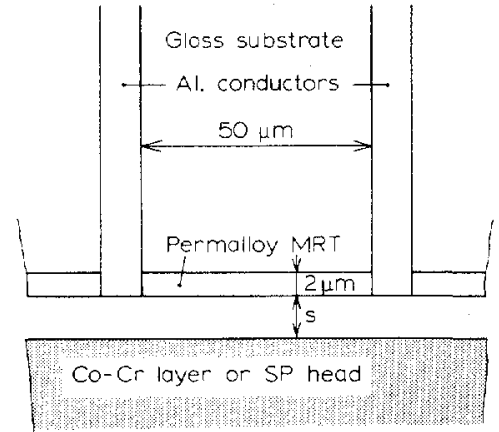

Fig. 3. MRT used for detection, placed at the edge of glass substrate at distance $s$ from $\mathrm{Co}-\mathrm{Cr}$ recording medium or SP head.

TABLE I

Physical Properties of Co-Cr Films Used

\begin{tabular}{|c|c|c|}
\hline FILM & A & B \\
\hline ats cr & 17.0 & 19.0 \\
\hline $\mathrm{E}=\quad(\mathrm{kA} / \mathrm{m})$ & 76 & 46 \\
\hline$M_{S} \quad(k A / n)$ & 675 & 450 \\
\hline $\mathrm{H}_{\mathrm{A}} \quad(\mathrm{kA} / \mathrm{m})$ & 560 & 240 \\
\hline s/1 & 0.04 & 0.05 \\
\hline$h(\mu m)$ & 1.00 & 1.07 \\
\hline$d(\mathrm{~lm})$ & 0.11 & 0.10 \\
\hline$\Delta \theta_{50}\left({ }^{\circ}\right)$ & 1.5 & 2.0 \\
\hline
\end{tabular}

$S_{\|}$is the in-plane remanence, $h$ the film thickness, $d$ the surface diameter of the crystallites, and $\Delta \theta_{50}$ the half width angle of the (002) rocking curve.

sensitive for its in-plane fields components because of the large demagnetization in the direction perpendicular to the film plane of the transducer. Because the MRT is a quadratic transducer by the very nature of the magnetoresistive effect, a bias field $H_{\text {bias }}$ is applied to control its sensitivity. The MRT output and displacement are registered on an $X Y$-recorder. For the stand-still recording experiments two $\mathrm{Co}-\mathrm{Cr}$ compositions were used. Their properties are listed in Table I.

The following features were examined:

- the congruence between calculated and measured MRT output,

- the performance of the SP head,

- the MRT read signal amplitude as a function of the head activation field $H_{0}$, and

- the medium noise, as could be detected by the MRT.

Because the MRT is able to detect static fields, medium noise is distinguished quite easily from other noise sources. A comparison was made between the medium noise of the two different compositions after dc-erasure.

\section{THEORY}

When a head field is applied to the recording medium, relation (2) can be written, as long as the recording medium is not saturated, as

$$
\left|H_{\text {tot }}\right|=\left|H_{0}+H_{\mathrm{SP}}(x, z)+H_{d}(y, z)\right| \leqslant H_{c} .
$$


$H_{\mathrm{SP}}$ is the vertical field component of the SP head, and $H_{0}$ its activating field. The presence of an activating field $H_{0}$ is quite essential for this type of head, in contrast with flux-closed heads, like the ordinary ring-type head and the head proposed for magnetic printing [8]: This field $H_{0}$ can be generated by means of an auxiliary pole [9], but for experimental ease we used a large coil surrounding the complete head and $\mathrm{Co}-\mathrm{Cr}$ medium. When recording, $H_{0}$ should be kept as small as possible with respect to $H_{\mathrm{SP}}$ (see further). In general the $H_{\mathrm{SP}} / H_{0^{-}}$ ratio $\left(=\mu_{m e}\right)$ will depend on the structure of the head. In this specific experiment, however, we are primarily interested in the nature of the reversals, induced by this type of head and not in its efficiency. For our type of heads $\mu_{m e}$ is about 20 (for details see Section IV and V-B).

In this section $H_{\mathrm{SP}}$ will be considered as known. In practice the measured head field is used (see Section IV). In general the fields $H_{\mathrm{SP}}$ and $H_{d}$ are not uniform and will exhibit $x$ - and $z$-components. However, as stated before, only the vertical components of the fields will be considered and therefore superscripts denoting the components are omitted.

According to the experimental procedure (see Section II) the medium is premagnetized negatively by a uniform saturation field and the SP head is activated by a positive field. Thus the resulting magnetization is given by the linear equation of the major hysteresis loop:

$$
H_{\text {tot }}=H_{0}+H_{\mathrm{SP}}(x, z)+H_{d}(x, z)=H_{c} .
$$

In this way nonlinearity due to the coercivity is avoided, greatly simplifying the problem.

In fact the value of $z$ must be specified, because in general the fields $H_{\mathrm{SP}}$ and $H_{d}$ have a different $z$-dependence. (See further.)

The initial condition of the medium is therefore the negative remanent state as shown in Fig. 1(a) for $H=0$, and the medium is magnetized by a positive head field yielding a local magnetization $M$, which is also determined by the local demagnetizing field $H_{d}$. Because of the latter, $M$ is not longer just proportional to $H_{\mathrm{SP}}$. When $H_{\mathrm{Sp}}$ becomes large enough, $M$ will change its sign. When the head field is switched off, $M$ will not change, as long as condition $(4 \mathrm{a})$ is satisfied, which then reduces to $\left|H_{d}\right| \leqslant H_{c}$.

The final magnetization and therefore $H_{d}$ too, is determined by $H_{\mathrm{SP}}$ as well as $H_{0}$. The MRT signal, however, is determined only by the nonuniform magnetization pattern, induced by $H_{\mathrm{SP}}$ (see further), and its transition amplitude. For this reason it is important to obtain a ratio of the amplitude of $H_{\mathrm{SP}}$ to $H_{0}$ that is as high as possible. This ratio depends, of course, on the head design and performance. For the type of SP head used it will be shown that the erasing effect of $H_{0}$ becomes quite appreciable for large values of $H_{0}$ (see Section V).

The general expression for the magnetic field, generated by divergence of the magnetization, reads

$$
\vec{H}_{1}=\int_{V} \frac{-(\nabla \cdot M)_{2} \vec{r}_{12}}{4 \pi r_{12}^{3}} d V_{2}
$$

The field $\vec{H}_{1}$ at a point 1 can be obtained by integration over the entire volume $V$ containing the magnetic "charge" density $-(\nabla \cdot M)$. The subscript 2 denotes the integration variable.
If only surface charge is assumed, the vertical field component $H(x, z)$ is derived as

$$
\begin{aligned}
H(x, z)= & \frac{1}{2 \pi} \int_{-\infty}^{+\infty} M\left(x^{\prime}\right) \frac{(z-h / 2)}{\left(x-x^{\prime}\right)^{2}+(z-h / 2)^{2}} d x^{\prime} \\
& -\frac{1}{2 \pi} \int_{-\infty}^{+\infty} M\left(x^{\prime}\right) \frac{(z+h / 2)}{\left(x-x^{\prime}\right)^{2}+(z+h / 2)^{2}} d x^{\prime}
\end{aligned}
$$

The first and second integrals are the contributions from the upper and lower film surface, respectively. The field outside the medium $(|z|>h / 2)$ is called the fringing field $H_{f}$ and inside the medium $(|z|<h / 2)$ the demagnetizing field $H_{d}$. It is easy to show that if $M$ is constant, $H_{f}$ equals zero, as should be expected for a uniform magnetized thin layer. Therefore the uniform change of $M$, caused by the uniform field $H_{0}$, will not contribute to the read-out signal. Inserting (6) into (4b) yields an integral equation of the first kind, that has to be solved for $M$. In fact the integrals in (6) are convolution integrals by the very nature of the kernel functions, i.e., the multiplicands of $M$ in the integrand. The Fourier transform of a function $f(x)$ is denoted by

$$
F[f(x)]=\hat{f}(\omega) .
$$

The Fourier transform of a convolution integral is given by [10]

$$
F\left[\int_{-\infty}^{+\infty} f\left(x^{\prime}\right) \cdot k\left(x-x^{\prime}\right) \cdot d x^{\prime}\right]=\hat{f}(\omega) \hat{k}(\omega)
$$

In this $k(x)$ is the kernel function. Application of this proposition to (6) yields [11]

$$
\begin{aligned}
& \hat{H}_{d}(\omega ; z)=\frac{-\hat{M}(\omega)}{2}\left\{e^{(z-h / 2) \omega}+e^{-(z+h / 2) \omega}\right\}, \\
& \text { for }|z|<\frac{h}{2}
\end{aligned}
$$

and

$$
\hat{H}_{f}(\omega ; z)=\frac{-\hat{M}(\omega)}{2} e^{\omega(z-h / 2)}\left(e^{-h \omega}-1\right), \text { for } z>h / 2 .
$$

Equation (9a) gives the Fourier transform of the demagnetizing field $H_{d}$ and (9b) of the fringing field $H_{f}$ above the film plane $z>h / 2$. Because $H_{\mathrm{SP}}$ is symmetrical in $x, M$ and $H_{d}$ behave likewise and the Fourier transform reduces to a cosine transform. Before solving (4b) we first separate the constant fields $H_{0}$ and $H_{c}$ from the head field $H_{\mathrm{SP}}$ which can be done by splitting $M$ into two parts:

$$
M=M_{1}+M_{2} \text {. }
$$

$M_{1}$ will be assigned to the uniform activating field:

$$
M_{1}=H_{c}-H_{0} \text {. }
$$

The same can be done for the nonuniform fields. Written in 


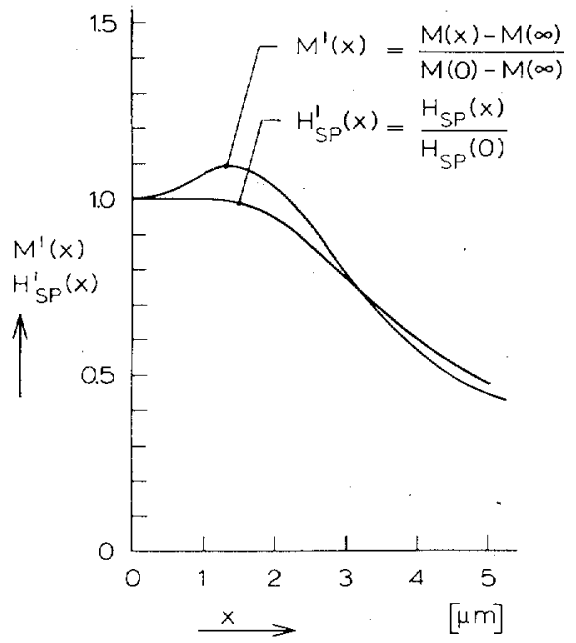

(a)

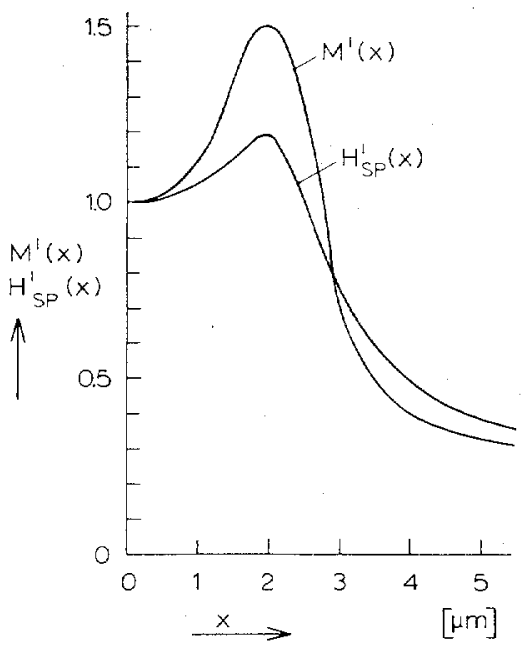

(b)

Fig. 4. Computed normalized magnetization $M$, induced by SP head field $H_{\mathrm{SP}}$, also normalized. $M$ is normalized with respect to $M(0)$ and $M(\infty)$.

the Fourier domain, it follows from (4b) that the demagnetizing field from $M_{2}$ is given by

$$
\hat{H}_{d}(\omega ; z)=-\hat{H}_{\mathrm{SP}}(\omega ; z)
$$

where the $z$-value denotes the point where, mathematically spoken, the switching takes place. It is noted here that $M_{2}$ is the nonuniform part of the magnetization which generates the read-out signal.

Next a choice has to be made for the value of $z$. As a first approximation the middle of the medium $z=0$ could be taken; then according to (9a) and (12) the cosine transform of $M_{2}$ is given by

$$
\hat{M}_{2}(\omega)=e^{h \omega / 2} \cdot \hat{H}_{\mathrm{SP}}(\omega ; 0) \text {. }
$$

A second approach is to take the fields, averaged over the film thickness. The average is obtained by integration of $(9 a)$ over the film thickness with respect to $z$ and dividing by $h$ :

$$
\hat{M}_{2}(\omega)=h \omega \hat{H}_{\mathrm{SP}}(\omega) /\left(1-e^{-h \omega}\right) \text {. }
$$

Here $H_{\mathrm{SP}}$ is averaged over the film thickness before calculating the cosine transform. The transformations are done numerically (see Appendix A).

Fig. 4 gives two examples of a calculation according to (13a). $H_{\mathrm{SP}}$ and $M$ are both normalized, the first with respect to $x=0$ and the latter to both $x=0$ and $x=\infty$, but because of the symmetry only half of the pattern is displayed. Clearly the magnetization increases strongly at the transition showing a peak (see Fig. 4(a)). This increase is enhanced if the head field itself shows a pronounced maximum at its edge (see Fig. 4(b)). The calculation according to (13b) gives only a slightly different result, as might be expected for the relative large width $(4.5 \mu \mathrm{m})$ of the SP head compared with the medium thickness $(1 \mu \mathrm{m})$ : the head field $H_{\mathrm{SP}}$, averaged over the film thickness, does not differ much from the head field in the middle of the film.

The pronounced peak in the magnetization at the transition is not the result of some peak in the head field (see Fig. 4(a)) but is only enhanced by such a peak (Fig. 4(b)). This advan- tageous feature must therefore be a result of the demagnetizing field. According to $4(\mathrm{~b})$, the relative demagnetizing and head fields are similar. This means that $H_{d}$ also decreases at the transition as $H_{\mathrm{SP}}$ does, resulting in the sharpening of $M$. This can be demonstrated more clearly in a schematical way, as shown by Suzuki and Iwasaki [12]. At a symmetrical single transition the vertical demagnetizing field goes through zero and this leads to the same type of sharpening as shown here by a more elaborate calculation. In practice transitions will not be symmetrical because of the asymmetrical nature of the head-field gradient.

\section{The Single Pole Head Field}

For the calculation of $\hat{M}(\omega)$ the head field must be known (13). In the experiments a single pole head has been used, because its vertical field component is suited better for perpendicular recording than that of traditional ring type heads [9] .

\section{A. Manufacturing of the SP Heads}

The SP heads were manufactured by means of RF sputtering from a Permalloy target with a bias voltage of $-100 \mathrm{~V}$ to suppress perpendicular anisotropy [13]. The easy axis $H_{c}$ is about $100 \mathrm{~A} / \mathrm{m}$, the intrinsic anisotropy field $H_{i}$ about 400 $\mathrm{A} / \mathrm{m}$, and the saturation magnetization $800 \mathrm{kA} / \mathrm{m}$. The easy axis is oriented along the $y$-axis and induced by means of a magnetic field of about $3 \mathrm{kA} / \mathrm{m}$ during sputtering.

\section{B. Head Field Measurements}

The head field $H_{\mathrm{SP}}$ in (13) is obtained by MRT measurements. For this purpose a SP head of relatively large thickness $(6.7 \mu \mathrm{m})$ was used. Its vertical head field component was measured with an MRT of $2-\mu \mathrm{m}$ width, which is relatively small compared with the head thickness. Therefore the head field gradients across the MRT will be small. As a consequence the head field may be assumed to be constant across the MRT in a first order approximation. This greatly facilitates the interpretation of the MRT responses as the MRT voltage can be 


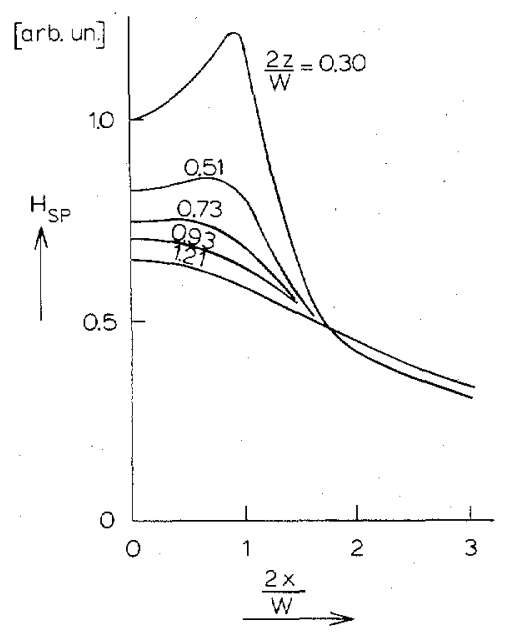

Fig. 5. Normalized head field measured on SP head 6.7- $\mu \mathrm{m}$ wide.

transformed directly into the magnetic field from the MRT calibration curve. The SP head field was measured at several separations between the MRT and the head.

In this way a two-dimensional rectangular grid of field values is obtained in the $x z$-plane. Now the MRT response can also be simulated on the basis of this measured field distribution. The $2-\mu \mathrm{m}$ MRT strip was thought to be divided into ten substrips of $0.2-\mu \mathrm{m}$ each to obtain a higher resolution and for each substrip the local head field was calculated by means of interpolation in the grid at the same $x z$-coordinates as in the measurements. Next the MRT response was calculated [14] and compared with the measured response which permits a refinement of the original field grid. In Fig. 5 the final result of the normalized head field is shown.

For small values of $z$ the head field exhibits a maximum at the edge of the head profile. At these $x z$-coordinates the field gradients are therefore large and especially here a refinement of the initial field grid is needed. Finally the agreement between the simulated and measured MRT response is better than 2 percent.

The scaled head field of Fig. 5 was used for the field calculation of the head actually used (having a thickness of $4.5 \mu \mathrm{m}$ ). The planar dimensions are about $15 \mathrm{~mm}$ square. Fig. 6 shows the measured and simulated MRT responses to be in excellent agreement.

\section{The Head Field Amplitude}

In principle the head field amplitude, defined as the field strength (as a function of $z$ ) at $x=0$, can also be obtained by means of MRT measurements as a function of the activating field $H_{0}$. The field range of the MRT, however, is limited by its saturation, which is determined by its effective anisotropy field. For the MRT used, this field was about $12 \mathrm{kA} / \mathrm{m}$. The amplitude in the recording experiments however far exceeds this limit.

Therefore the amplitude is determined by iteration, in the manner shown in Fig. 7 (this iteration is not included in the computer program). The amplitude is fitted to the measured MRT read-out signal. Of course this is only valid if no demag-

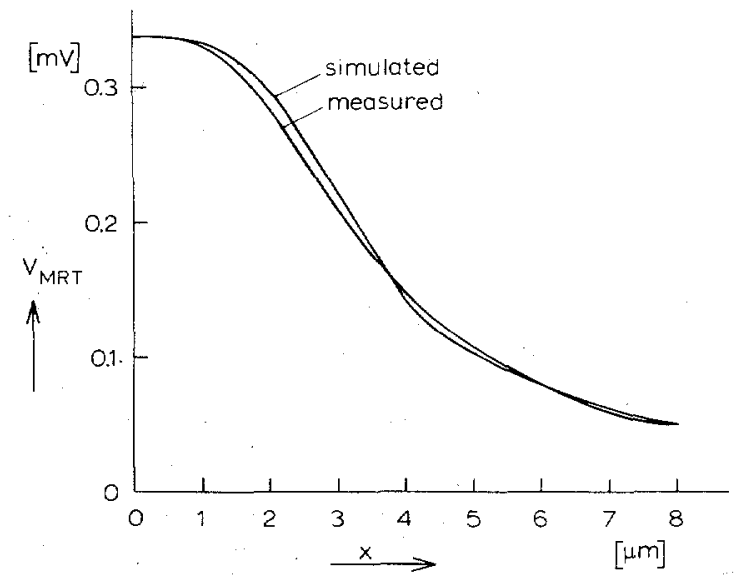

Fig. 6. Measured and simulated MRT response of $4.5 \mu \mathrm{m} \mathrm{SP}$ head field. Simulation is based on normalized field of Fig. 5 .

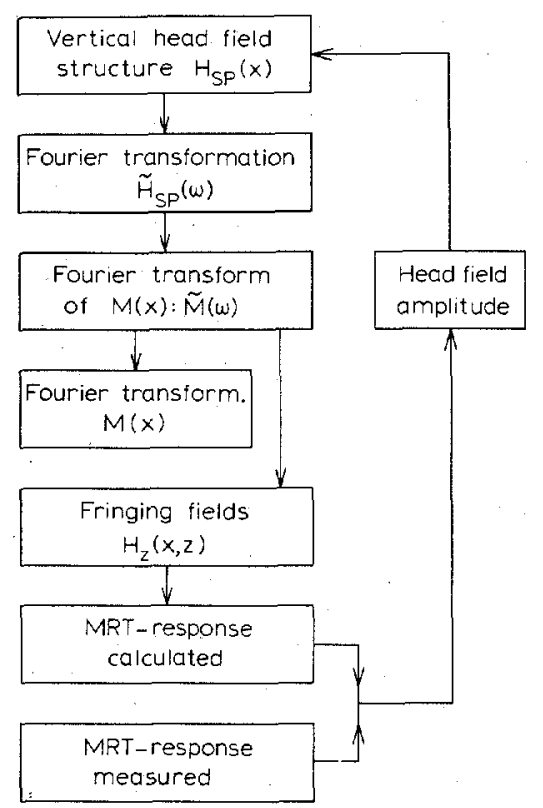

Fig. 7. Arithmetic procedure for calculation of MRI responses. Head field amplitude is determined by equalizing measured and calculated MRT output amplitude.

netization occurs when the head field is switched off. This point will be discussed later (see Section V).

\section{Head Field Permeability and Head Saturation}

Another complication might be the saturation of the head at larger activation fields, which is to be expected especially at the edges. This would result in a difference between the low field structure of Fig. 5 and the actual large field structure during recording. However no experimental evidence of saturation effects at the edges is found. Saturation of the head occurs mainly in the middle of the head because of the smaller local demagnetizing field. To clarify this point the head field permeability $\mu_{m e}$, defined as the ratio of the head field amplitude at $1 \mu \mathrm{m}$ distance, to the activating field $H_{0}$ [9], was determined.

This was done directly from the MRT head field measurements for small amplitudes of the head field and indirectly for 


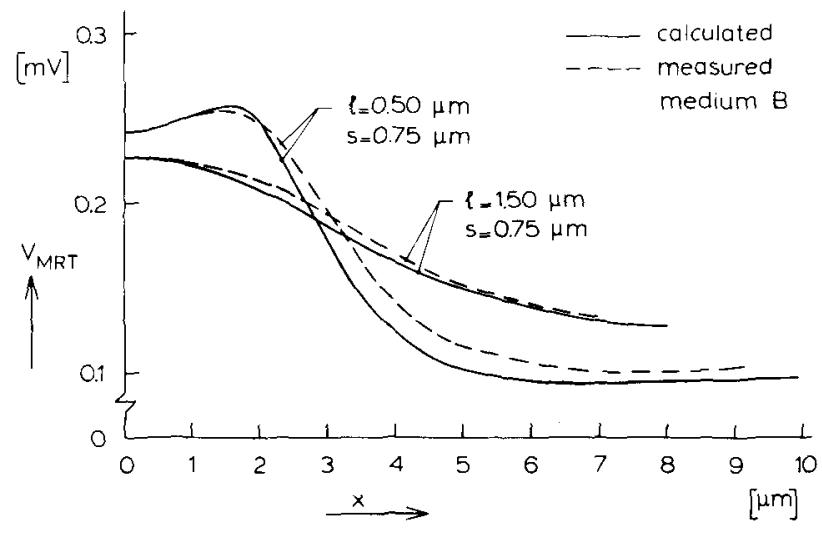

Fig. 8. Comparison of calculated and measured MRT responses for several separations between SP head and the medium; $l$ is head medium and $s$ the MRT medium separation.

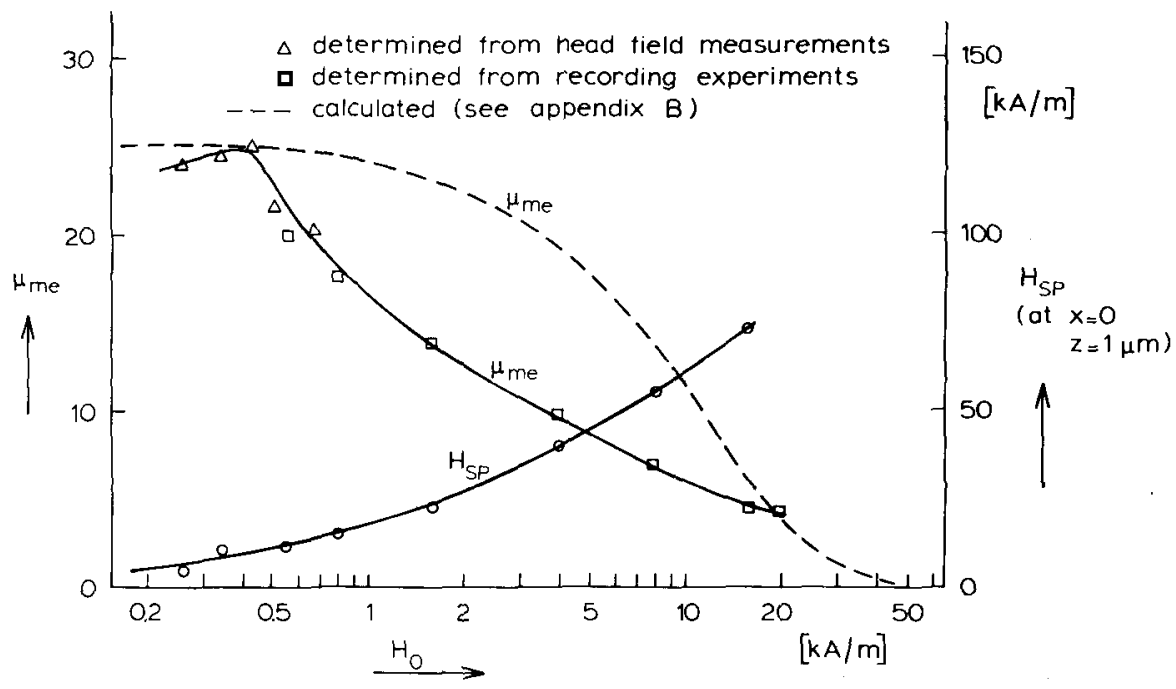

Fig. 9. Head field amplitude and its permeability $\mu_{m e}$ as a function of activating field $H_{0}$, defined at a distance of $1 \mu \mathrm{m}$ from the head at $x=0$.

larger amplitudes according to the computational scheme of Fig. 7. The results are compared with the ratio, calculated by means of a simple one-dimensional model (see Appendix B). The results will be discussed in the next section.

\section{Results and Discussion}

\section{A. Comparison between Calculated and Measured MRT-Output}

Fig. 8 shows two measured MRT signals together with the signals calculated by means of the procedure of Fig. 7 taking the averaged fields according to (13b). The signals are measured at two different distances between the MRT and the $\mathrm{Co}-\mathrm{Cr}$ medium. The agreement is satisfactory. However there is a reproducible horizontal shift of about $0.5 \mu \mathrm{m}$ between the simulated and measured MRT signals. The origin of this shift is not clear yet.

Although the MRT measurement of the head field did not exhibit a maximum in this case (see Fig. 6) the MRT signal of the induced transition does, demonstrating the expected influence of the demagnetizing field on the transition (see Fig. 4). A further confirmation of the model is found in Fig. 9. The indirect determination of the head field amplitude by means of the schema of Fig. 7 yields the same values as measured directly by means of the MRT in the low field range of $H_{0}$. For higher values of $H_{0}$ the head field amplitude can only be determined indirectly. To avoid ambiguity, this determination has been restricted to values of $\left|H_{d}\right|$ smaller than $H_{c} / 2$.

The main conclusions therefore are as follows.

1) The demagnetizing field has a favorable influence on the transitions in perpendicular stand-still recording.

2) The assumption that it is sufficient to consider only vertical components of the head field and the magnetization proved to be valid, due to the strong anisotropy field and the sharp perpendicular orientation of the $c$-axis.

3) The divergence of the magnetization may be considered to be restricted to the film surfaces. This is predominantly correct for the upper surface because it is closer to the MRT during reading.

Furthermore it may be concluded from Fig. 9 that there is no evidence of head-medium interactions, as might be expected [9]. However this point has not yet been investigated systematically and, therefore, this conclusion is restricted to the dimensions used in the experiment. 


\section{B. The SP Head Field}

Iwasaki and Nakamura [9] found a linear behavior of the SP head, if it is magnetized by an auxiliary pole placed opposite to the main pole. In this case the main pole is magnetized from its tip by the auxiliary pole field. If the intrinsic permeability of the head material is relatively large, the head field is determined by the local demagnetizing factor $N$ at the main pole tip only, resulting in a linear behavior. Because only the head tip is magnetized, it can be expected that the planar dimensions of the head are not relevant for the head performance.

Fig. 9 does not show a linear behavior of the type of SP head as used here. This is explained by the different manner of head activation; the head being activated by a homogeneous field and as a result it saturates for fields larger than the effective anisotropy field $H_{k}$, starting at the middle of the head (see Appendix B, Figs. 13 and 14).

The head performance is determined by the entire head volume. Fig. 9 shows a sharp decrease of $\mu_{m e}$ at $H_{0}=440 \mathrm{~A} / \mathrm{m}$, indicating saturation of the head. The value of $H_{k}$ at the middle of the head was measured directly by means of the magneto-optical Kerr effect. This yielded a value for $H_{k}$ of $545 \mathrm{~A} / \mathrm{m}$, which is somewhat larger then the former value. For the calculation of the head field permeability $\mu_{m e}$, as outlined in Appendix B, $H_{k}$ was taken as $440 \mathrm{~A} / \mathrm{m}\left(\mu_{m e}\right.$ is inversely proportional to $H_{k}$ ). The result is depicted by the dashed line in Fig. 9. As long as no saturation occurs the calculated and measure $\mu_{m e}$ are in good agreement. In the region of saturation however, the calculated $\mu_{m e}$ appears to be quite optimistic. The nonsaturation value $\mu_{m e}=25$ is much lower than the value of 150 found by Iwasaki and Nakamura [9] for a head of about the same pole thickness. This is probably caused by their more favorable method of head activation, i.e., pole tip activation.

\section{Signal Output Amplitude}

The MRT output voltage $V_{\text {MRT }}$ at $x=0$ is given in Fig. 10 as a function of the head activation field $H_{0}$. This was measured for the two different compositions used, 17 and 19 at $\%$ Cr with $H_{c} 76$ and $46 \mathrm{kA} / \mathrm{m}$, respectively (see Table I). For the range $H_{0}<8 \mathrm{kA} / \mathrm{m}$ there is no significant difference between the MRT output voltages. For a larger $H_{0}$, however, the MRT output of medium B saturates at a smaller value of $H_{0}$ than that of medium A. This is explained by the different coercivities of the media. Because the demagnetizing field is larger than the coercitive field in this range, demagnetizing takes place after switching off the head field. The maximum remanent magnetization or transition amplitude is then approximately determined by $\left|H_{d}\right|=H_{c}$. This equation however does not hold for the complete switch of $H_{\text {tot }}$ from $-H_{c}$ to $+H_{c}$ as can be seen in Fig. 1(a) of the perpendicular hysteresis loop.

Calculations show that in the case of demagnetization the corresponding $H_{d}$ equals about half the coercive field. The ratio of the maximum $H_{d}$ of the two compositions is about 0.5 , in the same order of magnitude as the ratio of their coercitive fields (=0.6). An important conclusion is, therefore, that the maximum remanent magnetization is proportional to

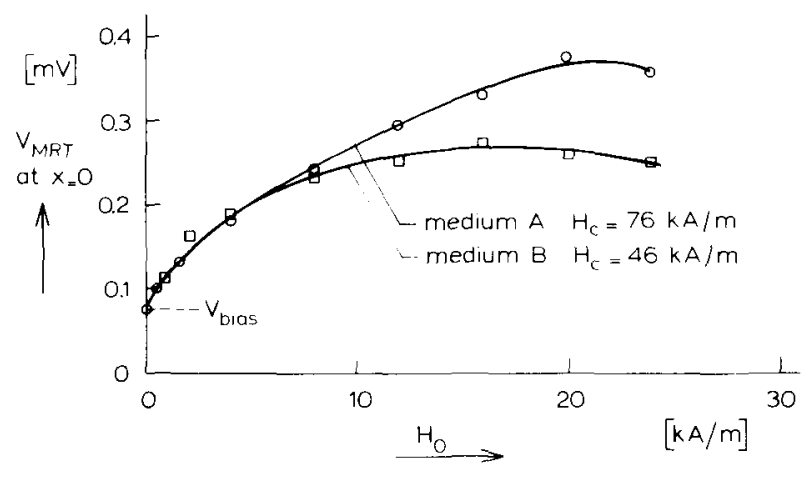

Fig. 10. MRT output as function of SP head activating field $H_{0}$ for two different $\mathrm{Co}-\mathrm{Cr}$ compositions used, i.e., 17 and 19 at $\% \mathrm{Cr}$.

$H_{c}$ and not related to $M_{s}$. This conclusion was already quite obvious from general considerations, concerning the perpendicular hysteresis loop (see Section II).

In the whole range of $H_{0}$, no significant change in the form of the MRT output signal is observed, not even in the case of demagnetization. This is an attendant advantage, because it means that the applicability of the proposed model is enhanced.

The MRT voltage amplitude as a function of $H_{0}$ shows a faint maximum for both compositions. This is caused by the fact that in this range the MRT output is limited by demagnetization of the $\mathrm{Co}-\mathrm{Cr}$ recording medium. Because the head field permeability $\mu_{m e}$ also strongly decreases in this range of $H_{0}$ (see Fig. 9), the erasing effect of $H_{0}$ becomes quite appreciable and the MRT output voltage, determined by the SP head field $H_{\mathrm{SP}}$ only, decreases for increasing $H_{0}$.

\section{The Remanent Magnetization}

Of course the experimental dimensions used, particularly the relative large pole thickness of $4.5 \mu \mathrm{m}$ compared with the film thickness of $1 \mu \mathrm{m}$, are not very favorable for reducing the demagnetization as might be expected for high-density recording. It is expected, however, that the demagnetization decreases for increasing bit density or decreasing main pole thickness. This is demonstrated by the following example. The remanent magnetization $M_{r}$ at $x=0$ was calculated for several values of the main pole thickness $W$. To facilitate the calculations a Gaussian head field is assumed:

$$
H_{\mathrm{SP}}(x)=A \cdot e^{-\left(2 x^{2} / W\right)} \text {. }
$$

The amplitude $A$ was kept constant in the calculations. It can be concluded from Fig. 11, that a considerable increase of the remanent magnetization is to be expected only if the bit distance is in the order of the film thickness or smaller.

\section{E. Medium Noise}

The noise from dc-erased films of both $\mathrm{Co}-\mathrm{Cr}$ compositions was also detected by means of the MRT. An example of this noise (from medium A) is shown in Fig. 12, together with a head print signal. Reversal of the direction in which the MRT was moved over the medium has no influence on the output as a function of position. Therefore the noise must be attributed to the medium. It should be noted that this noise is de- 


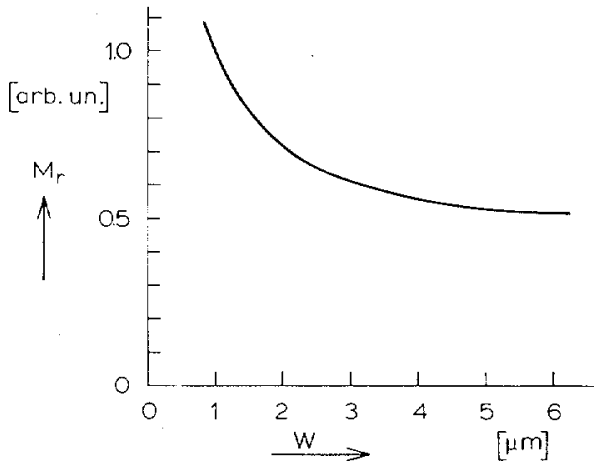

Fig. 11. Computed dependence of remanent magnetization $M_{r}$ at $x=$ 0 on width $W$ of writing field. For computational ease a Gaussian head field is assumed (see text).

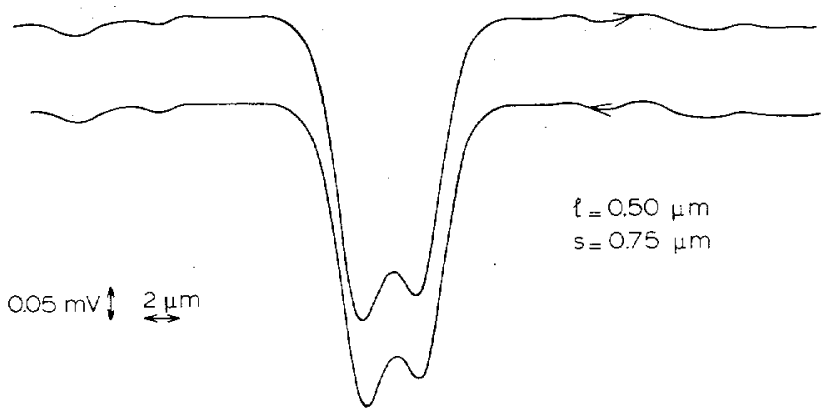

Fig. 12. Medium noise as detected by MRT from the 17 at $\% \mathrm{Cr}$ layer, together with stand-still recorded signal.

tected by a transducer that has a strong averaging character due to its length (i.e., trackwidth) of $50 \mu \mathrm{m}$. This means that a magnetic structure must be present which far exceeds the crystallyte dimensions in size. The Co-Cr medium noise has not been investigated systematically up until now, but it is shown [16] that noise may depend on the method of erasure. Nevertheless it was found that the ratio of the noise amplitudes of the different compositions is about equal to the ratio of their saturation magnetizations.

\section{APPENDIX A}

For simplification we assume that the head field $H_{\mathrm{SP}}$ equals zero outside a certain region:

$$
H_{\mathrm{SP}}(x)=0, \text { for }|x|>x_{m} .
$$

The Fourier transform is determined by a Fourier-series method [10]. $H_{\mathrm{SP}}$ is expanded as

$$
H_{\mathrm{SP}}(x)=\sum_{n=-\infty}^{+\infty} \alpha_{n} e^{j n \omega_{0} x}, \quad \text { for }|x|<x_{m}, \omega_{0}=\frac{\pi}{x_{m}}
$$

The coefficients $\alpha_{n}$ are given by

$$
\alpha_{n}=\frac{1}{2 x_{m}} \int_{-x_{m}}^{+x_{m}} H_{\mathrm{SP}}(x) \cdot e^{-j n \omega_{0} x} \cdot d x .
$$

Because of the symmetry of $H_{\mathrm{SP}}$ the problem reduces to a cosine transformation:

$$
H_{\mathrm{SP}}(x)=\alpha_{0}+2 \sum_{n=1}^{\infty} \alpha_{n} \cdot \cos \left(n \omega_{0} x\right)
$$

with

$$
\alpha_{n}=\frac{1}{x_{m}} \int_{0}^{x_{m}} H_{\mathrm{SP}}(x) \cdot \cos \left(n \omega_{0} x\right) \cdot d x .
$$

The problem is to determine the number $N_{m}$ at which series (18) can be truncated. This will depend on $H_{\mathrm{SP}}$. We have determined $N_{m}$ empirically. The cosine transform of $H_{\mathrm{SP}}$ is now given by $[10]$

$$
\begin{aligned}
\hat{H}_{\mathrm{SP}}(\omega)= & 2 x_{m} \alpha_{0} \frac{\sin \omega x_{m}}{\omega x_{m}} \\
& +\sum_{n=1}^{N_{m}} 2 x_{m} \alpha_{n}\left[\frac{\sin \left(\omega x_{m}-n \pi\right)}{\omega x_{m}-n \pi}+\frac{\sin \left(\omega x_{m}+n \pi\right)}{\omega x_{m}+n \pi}\right] .
\end{aligned}
$$

If the cosine transform of the kernel in (8) is denoted by $\hat{K}(\omega)$ then it follows with (12):

$$
\hat{M}(\omega)=\frac{\hat{H}_{\mathrm{SP}}(\omega)}{2 \hat{K}(\omega)} \text {. }
$$

As an example the case of $z=0$ is treated:

$$
\hat{M}(\omega)=e^{h \omega / 2} \cdot \hat{H}_{\mathrm{SP}}(\omega) \text {. }
$$

Here the problem of stability appears. In general (22) must obey

$$
\lim _{\omega \rightarrow \infty} \hat{M}(\omega)=0
$$

A practical approach is just to calculate the limit of $\hat{M}(\omega)$. For the head fields used (23) was always satisfied.

From (20) it is deduced

$$
\hat{M}(\omega)=0, \quad \text { for }|\omega|>\omega_{m}, \omega_{m}=\pi N_{m} / x_{m} .
$$

Now the magnetization $M$ can be determined following the same procedure and the fringing field also applying (9b).

\section{APPENDIX B}

In the calculation of a MRT response on a certain magnetic field, the magnetization of the strip is determined first and then the output voltage [7], [14], [17]. The same onedimensional model for calculating the magnetization is applied to the case of an SP-head [3]. Most calculations are numerical $[3],[7],[14]$ but under certain conditions an analytical expression is possible [17], greatly facilitating the calculation of the corresponding head field. The track-width of the SP head is assumed to be infinite. The easy axis of magnetization lies parallel to this infinite axis and is determined by demagnetization as well as intrinsic anisotropy (see Fig. 13). For the case that the intrinsic anisotropy field $H_{i}=0$ and in the onedimensional approximation it is derived by Tjaden [17]:

$$
\frac{M(z)}{M_{s}}=\frac{H_{0}}{H_{k}}\left[1-\left(\frac{2 z}{L}\right)^{2}\right]^{1 / 2}, \text { for }\left|H_{z}\right|<H_{k} .
$$

Herein is $H_{0}$ the uniform field, activating the SP head and $H_{k}$ the demagnetizing field, given by $M_{s} W / L$. W is the width and $L$ the length of the head. The point $z=0$ is at middle of the head. Numerically obtained results agree very well with this analytical expression (see Fig. 14(a)). Although (25) is derived 


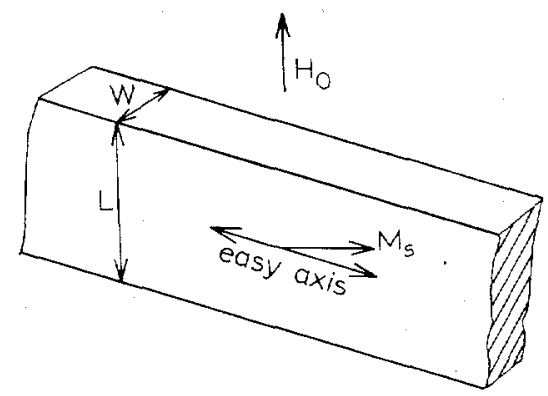

Fig. 13. Structure of SP head as used in one-dimensional calculation.

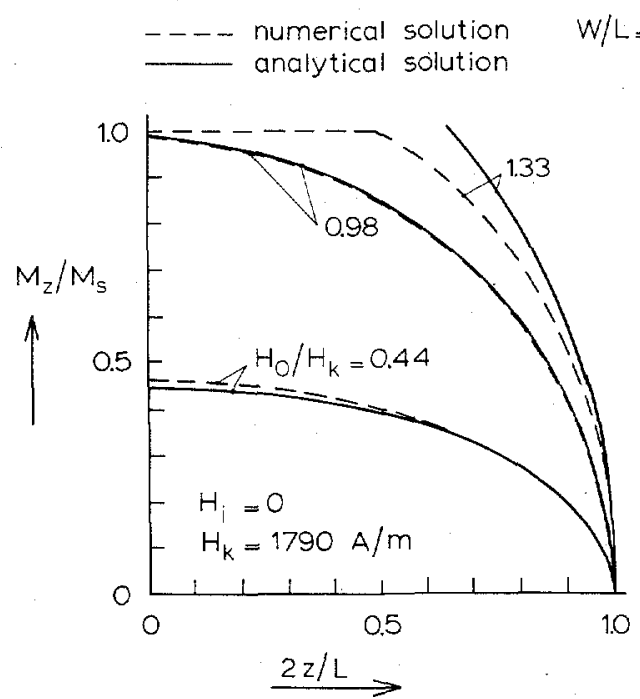

(a)

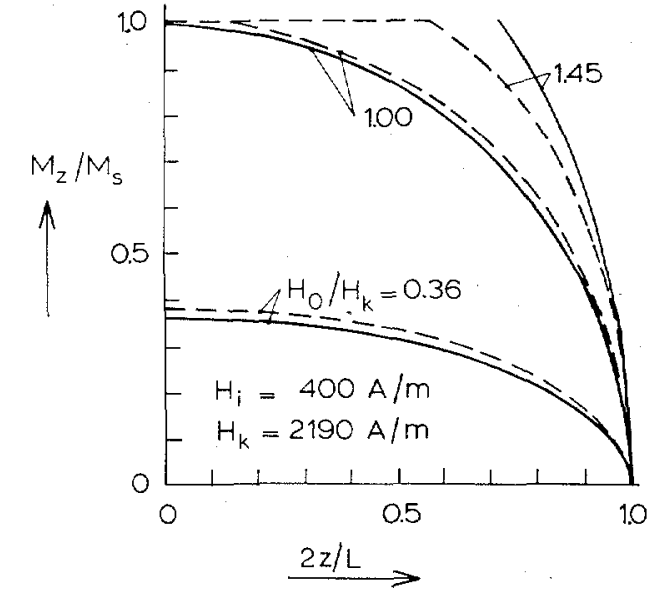

(b)

Fig. 14. Magnetization of SP head, activated by uniform field $H_{0}$. Head saturates first at middle. (a) Intrinsic anisotropy field $H_{i}=0$. (b) $H_{i}=400 \mathrm{~A} / \mathrm{m}$.

for $H_{i}=0$, good agreement is also obtained for $H_{i}>0$ if $H_{k}$ is replaced by $H_{k}=H_{i}+M_{s} W / L$ (see Fig. 14(b)). Furthermore if the head is saturating for $H_{0}>H_{k}$, the agreement between the numerical and analytical obtained magnetization is reasonable in the nonsaturated part of the head (see Fig. 14). For the case $H_{0} \gg H_{k}$, it is derived [17] :

$$
\frac{M(z)}{M_{s}}=\frac{2}{\pi}\{\arcsin \sqrt{s}+\sqrt{s}(1-s)\}
$$

for

$$
1-\frac{2 H_{k}}{\pi H_{0}}<\left|\frac{2 z}{L}\right|<1
$$

with

$$
s=\frac{\pi}{2} \frac{H_{0}}{H_{k}}\left(1-\left|\frac{2 z}{L}\right|\right) .
$$

After calculating the magnetization, the corresponding head field can be calculated. The head field $H_{\mathrm{SP}}(z)$ at $x=0$ is given by

$$
H_{\mathrm{SP}}(z)=\frac{1}{\pi} \int_{-L / 2}^{+L / 2} \frac{d M\left(z^{\prime}\right)}{d z^{\prime}} \arctan \left\{\frac{W}{2\left(z-z^{\prime}\right)}\right\} d z^{\prime}
$$

The derivative can be expelled by partial integration:

$$
H_{\mathrm{SP}}(z)=\frac{W}{2 \pi} \int_{-L / 2}^{+L / 2} M\left(z^{\prime}\right) \frac{d z^{\prime}}{\left(z^{\prime}-z\right)^{2}+(W / 2)^{2}} .
$$

This integral is evaluated numerically.

\section{ACKNOWLEDGMENT}

First we would like to thank A. van Herk and G. H. Jonker for their stimulating discussions. Further we are grateful to P. D. Ekkelkamp and A. J. F. Hollink for their assistance during the experiments and to B. Hurenkamp for the manufacture of the single pole heads. Finally we thank D. L. A. Tjaden for his permission to use some unpublished results.

\section{REFERENCES}

[1] A. van Herk and P. Wesseling, IEEE Trans. Magn., vol. MAG-10, no. 3, pp. 761-764, 1974.

[2] S. Iwasaki, Y. Nakamura, and K. Ouchi, IEEE Trans. Magn., vol. MAG-15, no. 6, pp. 1456-1458, 1979.

[3] J. Hokkyo, I. Saito, and S. Satake, IEEE Trans. Magn., vol. MAG16, no. 5 , pp. 887-889, 1980 .

[4] T. Wielinga and J. C. Lodder, IEEE Trans. Magn., vol. MAG-17, no. 6, pp. 3178 3180, 1981

[5] J. C. Lodder, T. Wielinga, and J. Worst, accepted for publication in Thin Solid Films.

[6] J. Worst, J. C. Lodder, and T. Wielinga, accepted for publication in Thin Solid Films.

[7] J. P. J. Groenland and J. H. J. Fluitman, J. Phys. E: Sci. Instrum., vol. 14 , pp. 503-508, 1981 
[8] J. P. Eltgen and J. G. Magnenet, IEEE Trans. Magn., vol. MAG16 , no. 5, pp. 961-966, 1980.

[9] S. Iwasaki and Y. Nakamura, IEEE Trans. Magn., vol. MAG-14, no. 5, pp. 436-438, 1978.

[10] A. Papoulis, The Fourier Integral and its Applications. New York: McGraw-Hill, 1962.

[11] H. Bateman, Tables of Integral Transforms. New York: McGrawHill, 1954.

[12] T. Suzuki and S. Iwasaki, IEEE Trans. Magn., vol. MAG-18, no. 2, pp. 769-771, 1982 .
[13] A. J. Collins, C. J. Prior, and R. C. Hicks, Perkin-Elmer seminar, Cambridge, England, 1981

[14] J. H. J. Fluitman, IEEE Trans. Magn., vol. MAG-14, no. 5, pp. 433-435, 1978.

[15] S. Iwasaki, K. Ouchi, and N. Honda, IEEE Trans. Magn., vol. MAG-16, no. 5, pp. 1111-1113, 1980.

[16] L. Thurlings, IEEE Trans. Magn., vol. MAG-16, no. 3, pp. 507513,1980 .

[17] D. L. A. Tjaden, private communication, Philips Research Laboratories, Eindhoven, The Netherlands.

\title{
NiFeCo-An Alternative to Permalloy for Bubble Domain Detection?
}

\author{
IAN L. SANDERS
}

\begin{abstract}
The characteristics of bubble domain sensors fabricated from ternary $\mathrm{NiFeCo}$ films have been studied and compared with Permalloy film sensors. In $350 \AA$ thick films, the ternary alloy exhibits a magnetoresistance ratio of 3.5 percent in contrast to 2.8 percent for Permalloy films prepared under similar conditions. Sensor sensitivity in functional bubble chips is correspondingly greater, while the sensor noise level is equal to-or lower than-that obtained with the Permalloy detector. Low coercivity and dispersion in NiFeCo films aid in producing an overall improvement in signal-to-noise ratio. The performance of $\mathrm{NiFeCo}$ sensors operating in $1-\mu \mathrm{m}$ bubble ion-implanted contiguous-disk devices is described.
\end{abstract}

\section{INTRODUCTION}

$\mathbf{M}$ AGNETORESISTIVE sensors for magnetic bubble device applications are routinely fabricated from binary $\mathrm{NiFe}$ alloys close the the nonmagnetostrictive $80: 20$ "Permalloy" composition. In the case of thick film detectors, the sensor material is governed primarily by the requirements of the bubble propagation structure, and there is little freedom to optimize the sensor composition for detection purposes. However the increasing use of thin film detectors, both in conventional Permalloy-bar [1] and ion-implanted contiguousdisk [2] devices has removed this constraint on the choice of sensor material.

In an early attempt to improve the signal sensitivity in thin film detectors, Asama et al. [3] investigated the use of binary alloys in the NiCo system. These materials are of interest because they can exhibit magnetoresistance ratios of up to 4

Manuscript received June 15, 1982; revised September 11, 1982. The paper was presented at the Third Joint INTERMAG-MMM Conference, Montreal, Quebec, Canada, July 20-23, 1982.

The author is with IBM Research Laboratory, Dep. K63/282, 5600 Cottle Road, San Jose, CA 95193. percent, compared with the typical 2-3 percent observed for Permalloy. Unfortunately the $\mathrm{NiCo}$ alloys have a relatively high coercivity $(\sim 15$ Oe in a $300 \AA$ film in contrast to $\sim 1.5$ Oe for Permalloy) and are strongly magnetostrictive. These factors give rise to considerable noise in the sensor output signal and restrict the practical usefulness of the material. It is important to remember that coercive force increases rapidly as the width of the sensor is reduced [4] and that small variations in sheet film coercivities are often amplified, rather than masked by the geometrical effect [5]. For example, although a sheet film may have a coercive force of 1-2 Oe, a 3- $\mu \mathrm{m}$ wide sensor etched from the same film can exhibit a coercivity of perhaps $20-30$ Oe. If the coercive force in the sensor becomes comparable in magnitude with the applied drive field $H_{x y}$, then erratic switching (i.e., noise) results. This is illustrated in Fig. 1, which shows the output from a 3- $\mu \mathrm{m}$ sensor with a coercive force of $60 \mathrm{Oe}$ operating in a 45-Oe rotating drive field. Here the amplitude of the switching noise is roughly half the amplitude of the bubble signal and is present over much of the rotating field cycle. The peak of the noise occurs as the rotating field sweeps past the long axis of the sensor where one expects incoherent domain-wall motion to take place.

Another requirement, not usually mentioned in the literature, is that the alloy should have a low dispersion of the easy-axis. If the dispersion in the film is low, the magnetization rotates coherently, while large dispersion on the other hand, leads to a nonretraceable magnetoresistance characteristic, and this in turn is another cause of poor detector signals. The effect of a dispersed easy-axis on the anisotropic magnetoresistance curve is illustrated in Fig. 2, comparing $300 \AA$ thick strips of $\mathrm{Ni}_{0.90} \mathrm{Fe}_{0.10}$ and $\mathrm{Ni}_{0.82} \mathrm{Fe}_{0.18}$ strips. Both films 